\title{
Neural Network based Handwritten Character Recognition system
}

\author{
Savitha Attigeri \\ M.Tech, Department of Computer Science and Engineering STJIT,Ranebennur
}

\begin{abstract}
:
Handwritten character recognition has been one of the active and challenging research areas in the field of image processing and pattern recognition. It has numerous applications which include, reading aid for blind, bank cheques and conversion of any hand written document into structural text form. In this paper an attempt is made to recognize handwritten characters for English alphabets without feature extraction using multilayer Feed Forward neural network. Each character data set contains 26 alphabets. Fifty different character data sets are used for training the neural network. The trained network is used for classification and recognition. In the proposed system, each character is resized into 30x20 pixels, which is directly subjected to training. That is, each resized character has 600 pixels and these pixels are taken as features for training the neural network. The results show that the proposed system yields good recognition rates which are comparable to that of feature extraction based schemes for handwritten character recognition.
\end{abstract}

\section{Introduction:}

Character recognition is an art of detecting, segmenting and identifying characters from image[1].An ultimate objective of hand written character recognition is to simulate the human reading capabilities so that the computer can read understand edit and work as human do with text[2]. Handwriting recognition has been one of the most fascinating and challenging research areas in field of image processing and pattern recognition in the recent years. It contributes immensely to the advancement of automation process and improves the interface between man and machine in numerous applications. Several research works have been focusing on new techniques and methods that would reduce the processing time while providing higher recognition accuracy. [3][4].Character recognition is mainly of two types online and offline. In online character recognition, data is captured during the writing process with the help of a special pen on electronic surface. In offline recognition, prewritten data generally written on a sheet of paper is scanned. Offline Character Recognition: Generally all printed or type-written characters are classified in offline mode. Off-line handwritten character recognition refers to the process of recognizing characters in a document that have been scanned from a surface such as a sheet of paper and are stored digitally in gray scale format. The storage of scanned documents have to be bulky in size and many processing applications as searching for a content, editing, maintenance are either hard or impossible. Online Character Recognition: The online mode of recognition is mostly used to recognize only handwritten characters. In this the handwriting is captured and stored in digital form via different means. Usually, a special pen is used in conjunction with an electronic surface. As the pen moves across the surface, the two- dimensional coordinates of successive points are represented as a function of time and are stored in order. Recently, due to increased use of handheld devices online handwritten recognition attracted attention of worldwide researchers. This online handwritten recognition aims to provide natural interface to users to type on screen by handwriting on a pad instead of by typing using keyboard. The online handwriting recognition has great potential to improve user and computer communication[5]. 
Several applications including mail sorting, bank processing, document reading and postal address recognition require offline handwriting recognition systems. As a result, the off-line handwriting recognition continues to be an active area for research towards exploring the newer techniques that would improve recognition accuracy[6][7].

\section{General Statement of the Problem Area}

The main objective of this research is to find a new solution for handwritten text recognition of different fonts and styles by improving the design structure of the traditional Artificial Neural Network (ANN). ANNs have been successfully applied to pattern recognition, association and classification, forecast studies, and control applications, to name a few. The recognition results of such text or handwritten materials are then fed into Optical Character Recognition (OCR) as an electronic translation of images of handwritten, typewritten or printed text into machine-editable text. OCR is a field of research that is fully developed and has been quite useful in pattern recognition, artificial intelligence and machine vision. Consequently, typewritten text recognition that is void of any distortions is now considered largely a solved problem. However the direct use of OCR on handwritten characters remains a very difficult problem to resolve, yielding extremely low reading accuracy. On-line handwritten character recognition involves the automatic conversion of text as it is written on a special digitizer or PDA, where a sensor picks up the pen-tip movements as well as pen-up and pen-down switching. The obtained signal is converted into letter codes, which are usable within computer and text-processing applications. Off-line handwritten document recognition is currently a difficult problem, as different people have different handwriting styles. Scanning, segmentation and classification are the general processes that are being used to recognize handwritten documents. In previous studies, ANNs have proven to be excellent recognizers of printed characters and handwritten digits (0 9), but research into recognition of handwritten words has not always been effective .

\section{The Proposed Recognition System}

In this section, the proposed recognition system is described. A typical handwriting recognition system consists of pre-processing, segmentation, classification and post processing stages. The general schematic diagram of the recognition system is shown in Fig.1.The proposed method which does not include feature extraction stage is shown in Fig1.

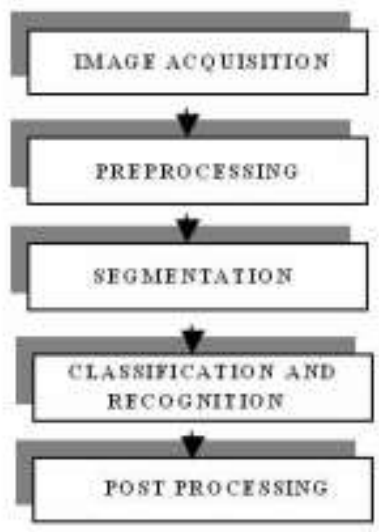

Fig 1. Schematic diagram of the proposed off-line recognition system

\section{Image acquisition}

In Image acquisition, the recognition system acquires a scanned image as an input image. The image should have a specific format such as JPEG, BMT etc. This image is acquired through a scanner, digital camera or any other suitable digital input device.

\section{Pre-processing}

The pre-processing is a series of operations performed on the scanned input image. It essentially enhances the image rendering it suitable for segmentation. The various tasks performed on the image in pre-processing stage are shown in Fig.2. Binarization process converts a gray scale image into a binary image using global thresholding technique. Dilation of edges in the binarized image is done using sobel technique, dilation the image and filling the holes present in it are the operations performed in the last two stages to produce the preprocessed image suitable for segmentation.

\section{Segmentation}

In the segmentation stage, an image of sequence of characters is decomposed into subimages of individual character In the proposed system, the pre-processed input image is segmented into isolated characters by assigning a number to each character using a labeling process. This 
labeling provides information about number of characters in the image. Each individual character is uniformly resized into $30 \mathrm{X} 20$ pixels.

\section{Classification and Recognition}

The classification stage is the decision making part of the recognition system . A feed forward back propagation neural network is used in this work for classifying and recognizing the handwritten characters. The 600 pixel derived from the resized character in the segmentation stage form the input to the classifier. The neural classifier consists of two hidden layers besides an input layer and an output layer as shown in Fig.4.The hidden layers use log sigmoid activation function and the output layer is a competitive layer as one of the characters is required to be identified at any point in time. The total number of neurons in the output layer is 26 as the proposed system is designed to recognize English alphabets .

\section{Post- processing}

Post-processing stage is the final stage of the proposed recognition system. It prints the corresponding recognized characters in the structured text form by calculating equivalent ASCII value using recognition index of the test samples.

\section{Pre-Processing Method:}

The raw data is subjected to a number of preliminary processing steps to make it usable in the descriptive stages of character analysis. Preprocessing aims to produce data that are easy for the OCR systems to operate accurately. Fig 3.1.1 shows the Pre-processing of handwritten character of image .The main objectives of pre-processing are :

- Noise reduction

- Binarization

- Edge detection

- Dilation and fillin

- Stroke width normalization

- Skew correction

- Slant removal

- Segmentation of processed image

\section{Noise Reduction}

When the document is scanned, the scanned images might be contaminated by additive noise and these low quality images will affect the next step of document processing. Therefore, a preprocessing step is required to improve the quality of images before sending them to subsequent stages of document processing. Due to the noise there can be the disconnected line segment, large gaps between the lines etc. so it is very essential to remove all of these errors so that's the information can be retrieved in the best way. There are many kinds of noise in images. One additive noise called "Salt and Pepper Noise", the black points and white points sprinkled all over an image, typically looks like salt and pepper, which can be found in almost all

documents. Noise reduction techniques can be categorized in two major groups as filtering, morphological operations.

\section{Filtering}

It aims to remove noise and diminish spurious points, usually introduced by uneven writing surface and/or poor sampling rate of the data acquisition device. Various spatial and frequency domain filters can be designed for this purpose

\section{Morphological Operations}

Morphological operations are commonly used as a tool in image processing for extracting image components that are useful in the representation and description of region shape. Morphological operations can be successfully used to remove the noise on the document images due to low quality of paper and ink, as well as erratic hand movement.

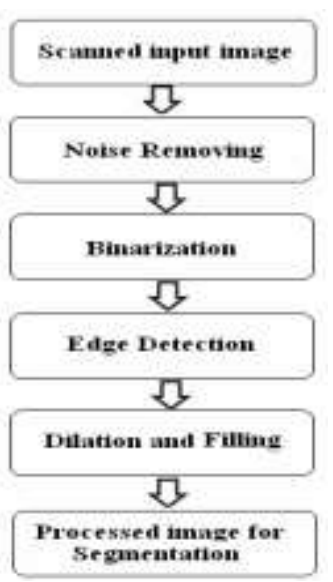

Fig.2 Pre-processing of handwritten character of image 


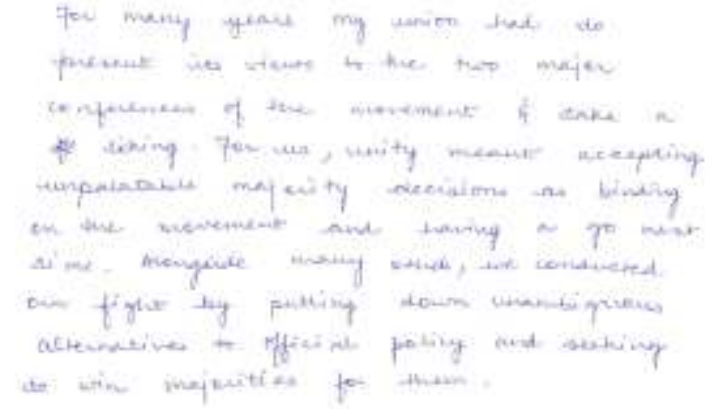

Fig.3

\section{Binarization}

Example for input scanned text image.

Binarization of gray-scale character images is a crucial step in offline character recognition. Good binarization facilitates segmentation and recognition of characters. Binarization process converts a gray scale image into a binary image. In this it has described new methods for the binarization of noisy gray-scale character images obtained in an industrial setting. Our methods are specially designed to binarize gray-scale character images more effectively by using the fact that characters are usually composed of thin lines of uniform width. Experimental results show that these methods give the best binarization results.

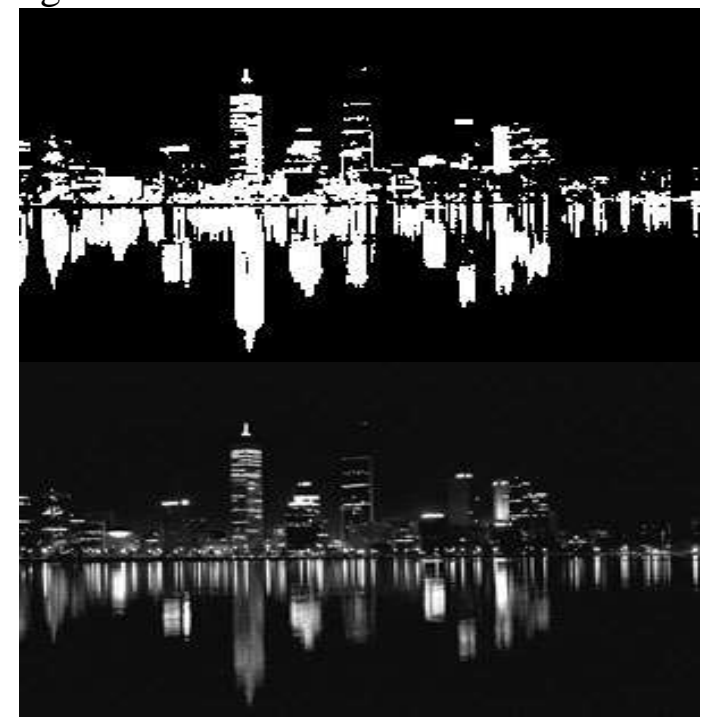

Fig.4 The example of binarization scheme.

\section{Edge Detection}

Edges characterize object boundaries and are therefore useful for segmentation, registration, and identification of objects. Edge detecting an image significantly reduces the amount of data and filters out useless information, while preserving the important structural properties in an image. There are many ways to perform edge detection. However, the majority of different methods may be grouped into two categories, gradient and Laplacian. The gradient method detects the edges by looking for the maximum and minimum in the first derivative of the image. The Laplacian method searches for zero crossings in the second derivative of the image to find edges.

\section{Thresholding}

In order to reduce storage requirements and to increase processing speed, it is often desirable to represent grey scale or color images as binary images by picking some threshold value for everything above that value is set to 1 and everything below is set to 0 . Two categories of thresholding exist: Global and Adaptive. Global thresholding picks one threshold value for the entire document image, often based on an estimation of the background level from the intensity histogram of the image. Adaptive thresholding is a method used for images in which different regions of the image may require different threshold values. A comparison of many common thresholding techniques is given by using an evaluation criterion that is goal-directed in the sense that the accuracies of a character recognition system using different techniques were compared. On those Tested, Niblack's method produced the best result.

\section{Dilation and fillin}

\section{a. Skew Detection}

For a document scanning process, there can be the skewness. There are several commonly used methods for detecting skew in a page; some rely on detecting connected components and finding the average angles connecting their centroids. The skewness should be removed because it reduces the accuracy of the document. The skew angle is calculated and with the help skew angle, the skewed lines are made horizontal. 


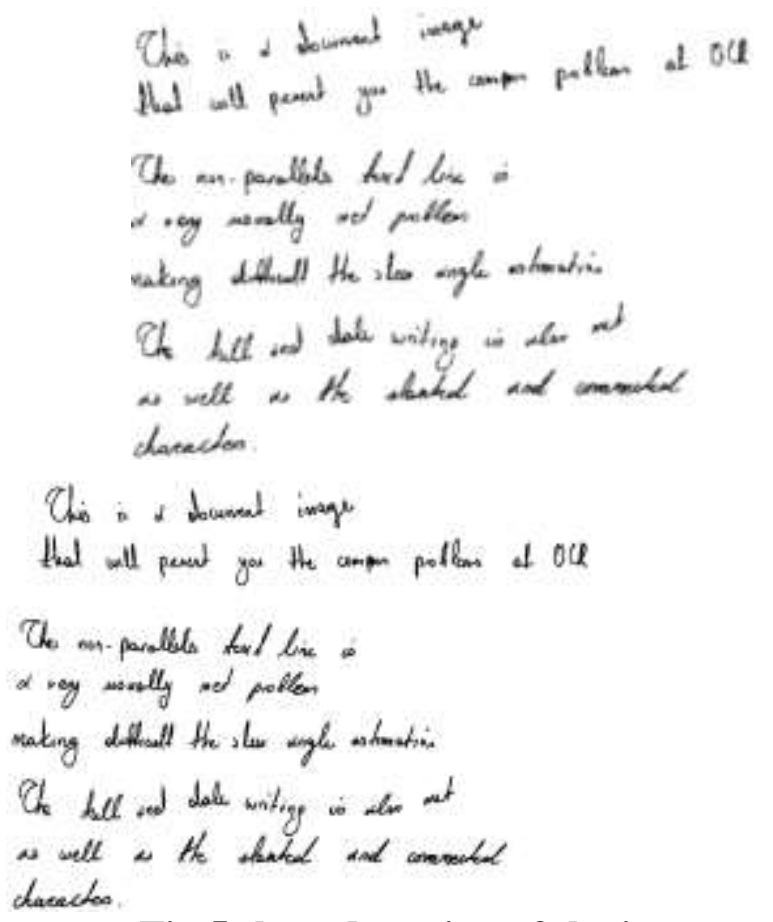

Fig.5 skew detection of the image.

\section{b. Slant Estimation and Normalization}

Handwritten text is usually characterized by slanted characters. In particular, the slanted characters slope either from right to left or vice versa. Moreover, different deviations may appear not only within a text but also within a single word. The slant correction does not affect the connectivity of the word and the resulting words are natural. Slant normalization is used to normalize all characters to a standard form. The most common method for slant estimation is the calculation of the average angle of near vertical elements. In this research, a slant removal algorithm is presented based on the use of the vertical projection profile of word images and the Wigner-Ville distribution. In this slant detection is performed by dividing the image into vertical and horizontal windows. The slant is estimated based on the center of gravity of the upper and lower half of each window averaged over all the windows. Another study has several methods have been proposed for average slant estimation and correction. However, average slant estimation has the problem such that local slant will be overestimated or underestimated when the slant in a word varies from character to character. To solve the problem, this paper proposes three methods for local slant estimation, which are simple iterative method, high speed iterative method and 8directional chain code method. The experimental results show that the proposed methods can estimate and correct local slant more accurately than the average slant correction. Lastly, a variant of Hough transform is used by scanning left to right across the image and calculating projections in the direction of 21 different slants. The top three projections for any slant are added and the slant with the largest count is taken as the slant value.

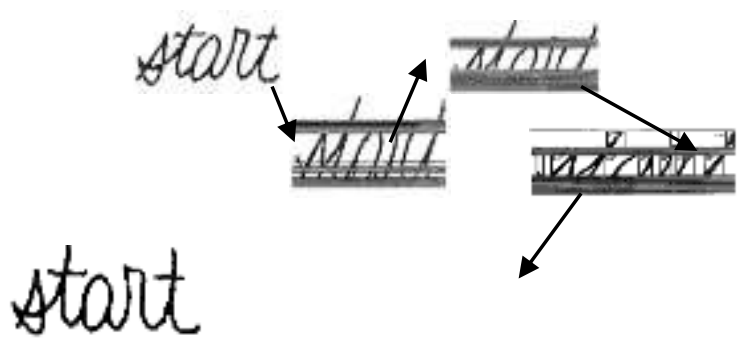

\section{E.SEGMENTATION}

Fig 6.slant removing example

In Character Recognition techniques, the Segmentation is the most important process. Segmentation is done to make the separation between the individual characters of an image. Segmentation of unconstrained handwritten word into different zones (upper middle and lower) and characters is more difficult than that of printed documents. This is mainly because of variability in inter-character distance, skew, slant, size and curved like handwriting. Sometimes components of two consecutive characters may be touched or overlapped and this situation complicates the segmentation task greatly. In Indian languages such touching or overlapping occurs frequently because of modified characters of upper-zone and lowerzone. Segmentation is an important stage, because the extent one can reach in separation of words, lines or characters directly affects the recognition rate of the script.

There are two types of segmentation:

\section{a) External Segmentation}

External segmentation decomposes the page layout into its logical units. External segmentation is the isolation of various writing units, such as paragraphs, sentences or words. It is the most critical part of document analysis. Document Analysis and Recognition (DAR) aims at the automatic extraction of information presented on paper and initially addressed to human comprehension. Segmenting the document image into text and non-text regions is an integral part of the OCR software. Therefore, one who works in the 
CR field should have a general overview for document analysis techniques. Page segmentation is one important step in layout analysis and is particularly difficult when dealing with complex layouts. Page layout analysis is accomplished in two stages: The first stage is the structural analysis, which is concerned with the segmentation of the image into blocks of document components (paragraph, row, word, etc). The second one is the functional analysis, which uses location, size and various layout rules to label the functional content of document components. Page segmentation is then implemented by finding textured regions in gray-scale or color images. For example, a method for automatically evaluating the quality of document page segmentation algorithms is introduced. They have proposed a bitmap-level automatic scheme to benchmark page segmentation algorithms on mixed text/halftone documents. It provides an accurate qualitative diagnosis of segmentation techniques, from which, a quantitative evaluation is derived.

\section{b) Internal Segmentation}

Internal Segmentation is an operation that seeks to decompose an image of a sequence of characters into sub images of individual symbols. Although, the methods have developed remarkably in the last decade and a variety of techniques have emerged, segmentation of cursive script into letters is still an unsolved problem. Character segmentation strategies are divided into three categories .

\section{Implementation On Graphical User Interface}

A user-friendly front end interface as shown in Fig.7 and Fig.8 has been implemented for the proposed handwritten character recognition system using menu based GUI (Graphical User Interface). The interface system presents the user with two menus - first menu with five processing stages and the second menu to choose the type feature extraction.

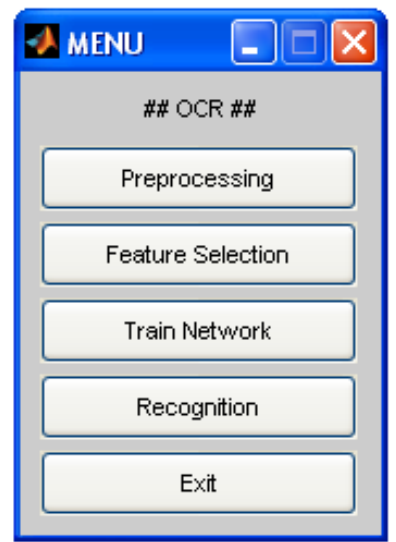

\section{Fig 7 Recognition System using Menu Based Graphical User Interface}

The menu based GUI enables the user to perform pre-processing, select the type of feature extraction, perform the feature extraction using the chosen method and train the network. After the network is trained, the recognition of the test image can be initiated by clicking the recognition bar on the interface. The test image is chosen using the facility provided for selecting the test images. Upon the completion of recognition process, the recognized image appears on the notepad. The same procedure can be used to recognize any number of test images. Finally the exit bar is used to quit from the character recognition system after recognizing all the test images. The GUI frees the user from the difficulties of working from the command line interface.

\section{Experimemntal Results}

The data were generate from 100 writers. Each writer wrote about 49 character by free-hand writing. The total data of 4840 characters were generate. The data were divided into two sets. The training set consists of 4400 and the test set is 440 data. Each character was preprocessing to Fourier extraction and the result of preprocessing is to recognition and identified by Back-Propagation Neural Network. The input layer is 10 nodes, hidden layer contained 70, 40, 30 nodes and output layer is 1 node. The network were performed was used in the test set process is $85.5 \%$. We train the system using the RCP training scheme to find the centroid of the prototype (44 Prototypes) and membership function. Finally, the FD of unknown character-image is used to perform recognition step. In this way the experimental results of recognition, 
RCP can perform with accuracy up to $91.5 \%$. the bolow figures shows some experimental examples:

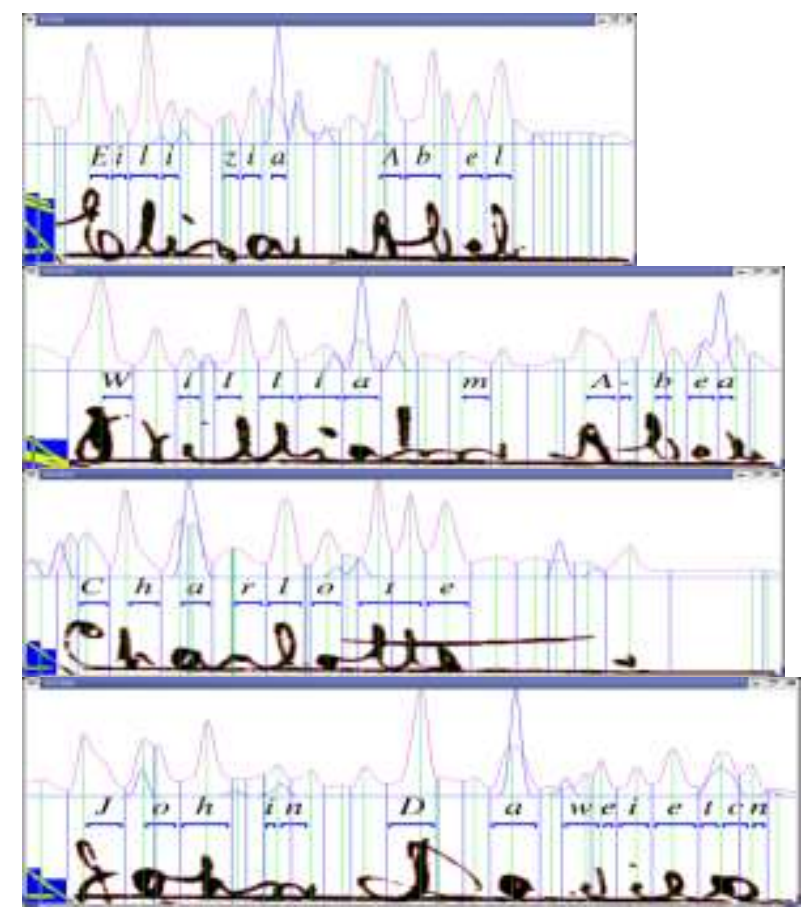

\section{CONCLUSIONS}

A neural network based off line handwritten character recognition system without feature extraction has been introduced in this paper for classifying and recognizing the 26 English alphabets. The pixel values derived from the resized characters of the segmentation stage have been directly used for training the neural network. As a result, the proposed

system will be less complex compared to the offline methods using feature extraction techniques. Of the several neural networks architectures used for classifying the characters, the one with two hidden layers each having 100 neurons has been found to yield the highest recognition accuracy of $90.19 \%$. The handwritten recognition system described in this paper will find potential applications in handwritten name recognition, document reading, conversion of any handwritten document into structural text form and postal address recognition.

\section{References}

[1] Mori, C.Y. Suen and K. Kamamoto, "Historical review of OCR research and development," Proc. of IEEE, vol. 80, pp. 1029-1058, July 1992.

[2] N. Arica and F. Yarman-Vural, "An Overview of Character Recognition Focused
onOff-line Handwriting", IEEE Transactions on Systems, Man, and Cybernetics, Part C: Applications and Reviews, Vol.31 (2), pp. 216 - 233. 2001.

[3] V.K. Govindan and A.P. Shivaprasad, "Character Recognition - A review," Pattern Recognition, Vol. 23, no. 7, pp. 671- 683, 1990.

[4] R. Plamondon and S. N. Srihari, "On-line and off- line handwritten character recognition: A comprehensive survey,"IEEE. Transactions on Pattern Analysis and Machine Intelligence, Vol. 22, no. 1, pp. 63-84,2000.

[5] U. Bhattacharya, and B. B. Chaudhuri, "Handwritten numeral databases of Indian scripts and multistage recognition of mixed numerals," IEEE Transaction on Pattern analysis and machine intelligence, Vol.31, No.3, pp.444-457, 2009.

[6] U. Pal, T. Wakabayashi and F. Kimura, "Handwritten numeral recognition of six popular scripts," Ninth International conference on Document Analysis and Recognition ICDAR 07, Vol.2, pp.749753, 2007.

[7] Pal, U. and B.B. Chaudhuri, "Indian script character recognition: A survey," Pattern Recognition, vol. 37,no.9, pp.1887-1899

[8] Mantas, J, "An overview of character recognition methodologies," Pattern Recognition, Vol. 19, Issue 6, pp. 425430, 1986.

[9] Rafael C. Gonzalez, Richard E. woods and Steven L.Eddins, Digital Image Processing using MATLAB, Pearson Education, Dorling Kindersley, South Asia, 2004.

[10] R.G. Casey and E.Lecolinet, "A Survey of Methods and Strategies in Character Segmentation," IEEE Transactions on Pattern Analysis and Machine Intelligence, Vol. 18, No.7, July 1996, pp. 690-706.

[11] A. Rajavelu, M. T. Musavi, and M. V. Shirvaikar, "A Neural Network Approach to Character Recognition," Neural Networks, 2, pp. 387-393, 1989.

[12] M. Hanmandlu, K.R.M. Mohan, and H. Kumar, "Neural-based Handwritten character recognition," in Proceedings of 
Fifth IEEE International Conference on Document Analysis and Recognition, ICDAR'99, pp. 241-244, Bangalore, India,1999. 